\title{
Single-spin asymmetry for forward neutron production
}

\author{
Jacques Soffer* \\ Department of Physics, Temple University, Philadelphia, PA 19122-6082, USA \\ E-mail: iacques.sofferdgmail.com
}

\section{B.Z. Kopeliovich}

Departamento de Física, Universidad Técnica Federico Santa María, Centro de Estudios Subatómicos, and Centro Científico-Tecnológico de Valparaíso, Casilla 110-V, Valparaíso, Chile

E-mail: boris.kopeliovicheusm.c1

\section{I.K. Potashnikova}

Departamento de Física, Universidad Técnica Federico Santa María, Centro de Estudios Subatómicos, and Centro Científico-Tecnológico de Valparaíso,

Casilla 110-V, Valparaíso, Chile

E-mail: irina.potashnikovadusm.c1

\section{Iván Schmidt}

Departamento de Física, Universidad Técnica Federico Santa María, Centro de Estudios Subatómicos, and Centro Científico-Tecnológico de Valparaíso, Casilla 110-V, Valparaíso, Chile

E-mail: ivan.schmidtausm.c]

The transverse single-spin asymmetry of neutrons produced at forward rapidities in polarized $p p$ collisions is calculated and compared with the recent measurements at RHIC. Absorptive corrections to the pion pole generating a relative phase between the spin-flip and non-flip amplitudes, lead to a transverse spin asymmetry, which however, is found to be far too small to explain the data. A larger contribution, comes from the interference of the pion and effective $\tilde{a}_{1}$-Reggeon, which includes the $a_{1}$ pole and the (dominant) $\pi \rho$ Regge cut. Assuming that this state saturates the spectral function of the axial current we determined its coupling to the nucleons applying the PCAC and the second Weinberg sum rule. The results of the parameter-free calculation of $A_{N}$ well agree with the data.

XXI International Workshop on Deep-Inelastic Scattering and Related Subject -DIS2013, 22-26 April 2013

Marseilles, France

\footnotetext{
*Speaker.
} 


\section{Pion pole corrected for absorption}

Polarization effects have always been known as a sensitive probe for interference between different contributions to the amplitude of the process. The single transverse spin asymmetry in reaction $p p \rightarrow n X$ with polarized protons was measured recently by the PHENIX experiment at RHIC [U] in $p p$ collisions at energies $\sqrt{s}=62,200$ and $500 \mathrm{GeV}$. The measurements were performed with a transversely polarized proton beam, and the neutron was detected at very forward and backward rapidities relative to the polarized beam. While no spin effects were detected in backward direction ${ }^{1}$, an appreciable single transverse spin asymmetry was observed in events with large fractional neutron momenta $z$. The data agree with a linear dependence on the neutron transverse momentum $q_{T}$, and different energy match well, what indicates at an energy independent $A_{N}\left(q_{T}\right)$.

Pion exchange is a natural candidate for the dominant mechanism of leading neutron production. In Born approximation for pion exchange depicted in Fig. $\mathbf{W}$ (left), the amplitude has the form [B],

$$
A_{p \rightarrow n}^{B}(\vec{q}, z)=\bar{\xi}_{n}\left[\sigma_{3} q_{L}+\frac{1}{\sqrt{z}} \vec{\sigma} \cdot \vec{q}_{T}\right] \xi_{p} \phi^{B}\left(q_{T}, z\right),
$$

where $\vec{\sigma}$ are the Pauli matrices; $\xi_{p, n}$ are the proton or neutron spinors; $\vec{q}_{T}$ and $q_{L}=m_{N}(1-z) / \sqrt{z}$, are the transverse and longitudinal components of the momentum transfer respectively. At large $z$ the amplitude $\phi^{B}\left(q_{T}, z\right)$ has the Regge form [四],

$$
\phi^{B}\left(q_{T}, z\right)=\frac{\alpha_{\pi}^{\prime}}{8} G_{\pi^{+} p n}(t) \eta_{\pi}(t)(1-z)^{-\alpha_{\pi}(t)} A_{\pi^{+} p \rightarrow X}\left(M_{X}^{2}\right),
$$

where $M_{X}^{2}=(1-z) s ;-t=q_{L}^{2}+q_{T}^{2} / z$. Both the spin-flip and non-flip amplitudes in (ㅁ. $\mathbb{C}$ ) have the same phase, given by the signature factor, $\eta_{\pi}(t)=i-\cot \left[(\pi / 2) \alpha_{\pi}(t)\right]$, so they do not produce any single-spin asymmetry.

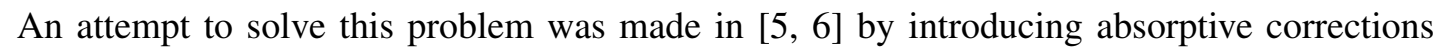
corresponding to initial/final state interactions of the projectile partons. However, the calculated phase shift between the spin-flip and non-flip amplitudes was found to be too small to explain the PHENIX data on $A_{N}$. The resulting asymmetry is depicted in Fig. ( (right).

\section{Axial-vector Reggeons and Regge cuts}

In addition to pion exchange, other Regge poles $R=\rho, a_{2}, \omega, a_{1}$, etc. and Regge cuts can contribute to the $p p \rightarrow n X$ reaction as is illustrated graphically in Fig. $\square$ (left).

Summing over different produced states $X$ and using completeness one arrives at the imaginary part of the amplitude of the process $\pi+p \rightarrow R+p$ at c.m. energy $M_{X}^{2}$. The production of natural parity states, like $\rho, a_{2}$, etc. can proceed only via Reggeon exchange, therefore these amplitudes are strongly suppressed at RHIC energies by a power of $M_{X}$ (dependent on the Regge intercept) and

\footnotetext{
${ }^{1}$ Notice that observation of no spin effects at large negative rapidities [四] can be explained by the so called Abarbanell-Gross theorem [■], which states that the single-spin asymmetry at this kinematic region should be exactly zero within the Regge-pole approximation.
} 

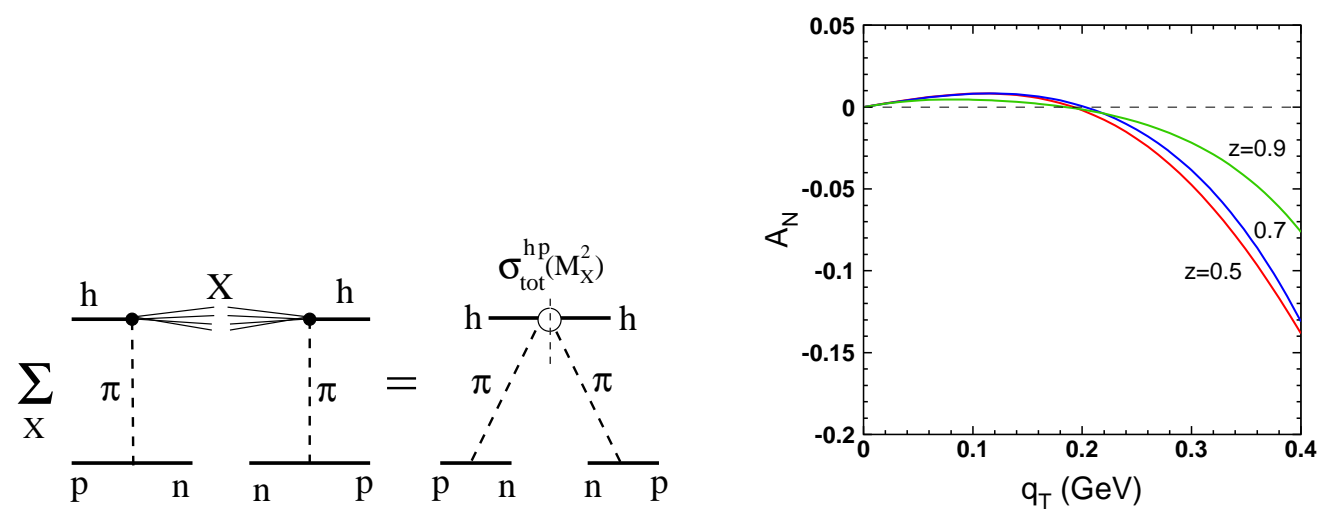

Figure 1: Left: graphical representation of the cross section of inclusive neutron production in hadronproton colisions, in the fragmentation region of the proton. Right: Single transverse spin asymmetry of leading neutrons related to the single pion exchange corrected for absorptive corrections, as function of $q_{T}$. The curves from bottom to top correspond to $z=0.5,0.7$ and 0.9

can be safely neglected everywhere, except the region of very small $(1-z) \sim s_{0} / s$, unreachable experimentally.

Only the unnatural parity states, which can be diffractively produced by a pion, like the $a_{1}$ meson, or $\rho-\pi$ in the axial vector or pseudo-scalar states, contribute to the interference term in the neutron production cross section at high energies.

The $a_{1} N N$ vertex is pure non spin-flip [焑], therefore, it should be added to the first term in Eq. (ㅁ. (ل),

$$
A_{p \rightarrow n}^{a_{1}}\left(q_{T}, z\right)=e_{\mu}^{L} \bar{n} \gamma_{5} \gamma_{\mu} p=\frac{2 m_{N} q_{L}}{\sqrt{|t|}} \phi_{0}^{a_{1}}\left(q_{T}, z\right) \bar{\xi}_{n} \sigma_{3} \xi_{p}
$$

where the longitudinal polarization vector of $a_{1}$ reads [ []], $e_{\mu}^{L}=\left(\sqrt{q_{0}^{2}-t}, 0,0, q_{0}\right) / \sqrt{|t|}$, and the transferred energy $q_{0}=E_{p}-E_{n}=q_{L}+O\left(m_{N} / \sqrt{s}\right)$. In the Born approximation,

$$
\phi_{0}^{a_{1}}\left(q_{T}, z\right)=\frac{\alpha_{a_{1}}^{\prime}}{8} G_{a^{+} p n}(t) \eta_{a_{1}}(t)(1-z)^{-\alpha_{a_{1}}(t)} A_{a_{1}^{+} p \rightarrow X}\left(M_{X}^{2}\right),
$$

and $\eta_{a_{1}}(t)=-i-\tan \left[(\pi / 2) \alpha_{a_{1}}(t)\right]$.

The amplitude (․] contains three unknowns, which we fix as follows.

The amplitude $A_{a_{1}^{+} p \rightarrow X}\left(M_{X}^{2}\right)$ is normalized as,

$$
\sum_{X} A_{a_{1}^{+} p \rightarrow X}^{\dagger}\left(M_{X}^{2}\right) A_{\pi p \rightarrow X}\left(M_{X}^{2}\right)=4 \sqrt{\pi} M_{X}^{2} \sqrt{d \sigma\left(\pi p \rightarrow a_{1} p\right) /\left.d p_{T}^{2}\right|_{p_{T}=0}}
$$

The $a_{1}$ pole is very weak, it has been observed in $\pi \rightarrow 3 \pi$ diffraction only by means of a phase-shift analysis [ [8]. A much large contribution comes from the axial-vector state $\rho \pi\left(1^{+} S\right)$, which has the invariant mass distribution forming a strong and narrow peak at $M_{\pi \rho} \approx 1.1 \mathrm{GeV}$. So, we introduce and employ in what follows the effective "pole" $\tilde{a}_{1}$ in the dispersion relation for the axial-vector current, and predict its production cross section in $\pi p$ collisions at high energies. The cross section $d \sigma\left(\pi p \rightarrow \tilde{a}_{1} p\right) /\left.d p_{T}^{2}\right|_{p_{T}=0}$ was fitted to data [ [ $]$ ]. 
The $\tilde{a}_{1}$-nucleon vertex $G_{\tilde{a}_{1} N N}(t)$ was related in [目] to the $\pi$-NN vertex basing on the PCAC relation,

$$
\frac{\sqrt{2} f_{\tilde{a}_{1}} g_{\tilde{a}_{1} N N}}{m_{\tilde{a}_{1}}^{2}}=\frac{f_{\pi} g_{\pi N N}}{\sqrt{2} m_{N}}
$$

where $f_{\tilde{a}_{1}}=f_{\rho}=\sqrt{2} m_{\rho}^{2} / \gamma_{\rho}$ according to the second Weinberg sum rule.

The Regge trajectory of the effective $\tilde{a}_{1}$-pole is given by the trajectory of the $\rho$ - $\pi$ Regge cut,

$$
\alpha_{\tilde{a}_{1}}(t)=\alpha_{\pi \rho}(t)=\alpha_{\pi}(0)+\alpha_{\rho}(0)-1+\frac{\alpha_{\pi}^{\prime} \alpha_{\rho}^{\prime}}{\alpha_{\pi}^{\prime}+\alpha_{\rho}^{\prime}} t .
$$

Eventually, we are in a position to perform a parameter free calculation of the $\tilde{a}_{1}-\pi$ interference contribution to the single transverse spin asymmetry of neutron production,

$$
\begin{aligned}
A_{N}\left(q_{T}, z\right) & =q_{T} \frac{4 m_{N} q_{L}}{|t|^{3 / 2}}(1-z)^{\Delta \alpha(t)} \frac{\operatorname{Im} \eta_{\pi}^{*}(t) \eta_{\tilde{a}_{1}}(t)}{\left|\eta_{\pi}(t)\right|^{2}} \\
& \times \frac{g_{\tilde{a}_{1}^{+} p n}}{g_{\pi^{+} p n}}\left(\frac{d \sigma_{\pi p \rightarrow \tilde{a}_{1} p}\left(M_{X}^{2}\right) /\left.d p_{T}^{2}\right|_{p_{T}=0}}{d \sigma_{\pi p \rightarrow \pi_{p}}\left(M_{X}^{2}\right) /\left.d p_{T}^{2}\right|_{p_{T}=0}}\right)^{1 / 2},
\end{aligned}
$$

where $\Delta \alpha(t)=\alpha_{\pi}(t)-\alpha_{\tilde{a}_{1}}(t)$. The results of calculations for every value of $z$ corresponding to the experimental point, are plotted by asterisks in Fig. $\square$ (right). They agree well with the PHENIX data.
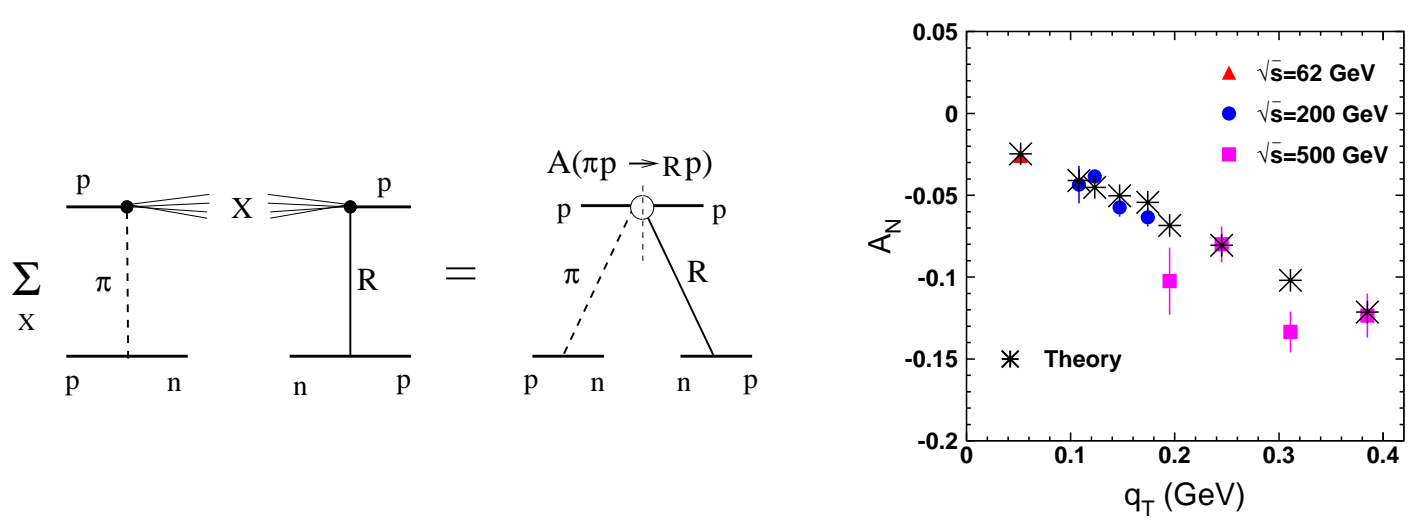

Figure 2: Left: graphical representation for the interference between the amplitudes with pion and Reggeon exchanges. Right: single transverse spin asymmetry $A_{N}$ in the reaction $p p \rightarrow n X$, measured at $\sqrt{s}=62,200,500 \mathrm{GeV}$ [四]. The asterisks show the result of calculation with Eq. ([2.6), which was done point by point, since each experimental point has a specific value of $z$.

\section{Summary}

Although some cross section data of leading neutron production in $p p$ collisions at high energies are well explained by the pion pole exchange supplemented with (significant) absorptive corrections $^{2}$, this description fails to reproduce the magnitude of the transverse single-spin asymmetry in polarized $p p$ collisions, measured recently by the PHENIX collaboration at RHIC.

${ }^{2}$ The PHENIX collaboration has recently released the integrated cross section at $\sqrt{s}=200 \mathrm{GeV}$, with large errors [0]], in agreement with an earlier measurement at ISR [Q] , which overestimates the normalization. Note that an excellent description of the DIS data has been published recently [प]]. 
Another possible source of spin effects is the interference between the amplitudes of neutron production via pion and $a_{1}$ Reggeon exchanges. Because $a_{1}$ has unnatural parity, it can be produced diffractively in $\pi+p \rightarrow a_{1}+p$, so is not suppressed at high c.m. energy $M_{X}$. It also provides a large, close to maximal, relative phase shift between the non-flip $a_{1}$ and spin-flip pion exchange amplitudes.

It turns out, however, that the $a_{1}$ exchange contribution is strongly suppressed by the smallness of the diffractive $a_{1}$ resonance production. Nevertheless, we found that it is possible to replace this resonance by $\pi \rho$ in the unnatural parity $1^{+} S$ state, because it forms a narrow peak in the $3 \pi$ invariant mass distribution, so can be treated as an effective pole, named $\tilde{a}_{1}$, in the dispersion relation for the axial current.

Presence of such an effective pole in the dispersion relation for the axial current allows to determine the $\tilde{a}_{1}$-nucleon coupling using PCAC, which relates the contributions of heavy states (saturated by the $\tilde{a}_{1}$ pole and the pion pole). Additional information about the leptonic decay constant of $\tilde{a}_{1}$ is obtained from the second Weinberg sum rule.

Although the $\pi \rho$ exchange corresponds to a Regge cut, rather than a pole, we found its Regge intercept to be rather close to the one for $a_{1}$ Reggeon, so the phase shift is similar as well.

Eventually, we calculated the single transverse spin asymmetry at different values of the kinematic variables, $s, q_{T}$ and $z$, and found very good agreement with data with no adjustment of parameters.

We have found a simple mechanism to describe the single-spin asymmetry data. It might be useful to investigate it at higher $q_{T}$, in view of future experimental data.

This work was supported in part by Fondecyt (Chile) grants 1090291, 1090236 and 1100287.

\section{References}

[1] K. Tanida [PHENIX Collaboration], J. Phys. Conf. Ser. 295, 012097 (2011).

[2] H. D. I. Abarbanel and D. J. Gross, Phys. Rev. Lett. 26, 732 (1971); Phys. Rev. D 5, 699 (1972).

[3] B. Z. Kopeliovich, I. K. Potashnikova, I. Schmidt and J. Soffer, Phys. Rev. D 78, 014031 (2008).

[4] B. Kopeliovich, B. Povh and I. Potashnikova, Z. Phys. C 73, 125 (1996).

[5] B. Z. Kopeliovich, I. K. Potashnikova, I. Schmidt and J. Soffer, AIP Conf. Proc. 1056, 199 (2008).

[6] B. Z. Kopeliovich, I. K. Potashnikova, I. Schmidt and J. Soffer, Phys. Rev. D 84, 114012 (2011).

[7] B. Z. Kopeliovich and P. Marage, Int. J. Mod. Phys. A 8, 1513 (1993).

[8] Yu. M. Antipov et al., Nucl. Phys. B 63, 153 (1973) [Erratum-ibid. B 76, 574 (1974)].

[9] W. Flauger and F. Mönnig, Nucl. Phys. B 109, 347 (1976).

[10] A. Adare et al., [PHENIX Collaboration], arXiv: 1209.3283 [nucl-ex].

[11] B. Z. Kopeliovich, I. K. Potashnikova, B. Povh and I. Schmidt, Phys. Rev. D 85, 114025 (2012). 